OPEN ACCESS

Edited by:

Detlev Boison,

Legacy Health, United States

Reviewed by:

Beena Pillai,

Institute of Genomics and Integrative

Biology (CSIR), India

Eva Maria Jimenez-Mateos,

Royal College of Surgeons in Ireland,

Ireland

*Correspondence:

Tamara Roitbak

troitbak@salud.unm.edu

Received: 08 December 2017 Accepted: 12 February 2018

Published: 23 February 2018

Citation:

Roitbak T (2018) Silencing a Multifunctional microRNA is Beneficial for Stroke Recovery.

Front. Mol. Neurosci. 11:58. doi: 10.3389/fnmol.2018.00058

\section{Silencing a Multifunctional microRNA Is Beneficial for Stroke Recovery}

\author{
Tamara Roitbak* \\ Department of Neurosurgery, Health Sciences Center, University of New Mexico, Albuquerque, NM, United States
}

Stroke-induced endothelial cell injury leads to destruction of cerebral microvasculature and significant damage to the brain tissue. A subacute phase of cerebral ischemia is associated with regeneration involving the activation of vascular remodeling, neuroplasticity, neurogenesis, and neuroinflammation processes. Effective restoration and improvement of blood supply to the damaged brain tissue offers a potential therapy for stroke. microRNAs (miRNAs) are recently identified small RNA molecules that regulate gene expression and significantly influence the essential cellular processes associated with brain repair following stroke. A number of specific miRNAs are implicated in regulating the development and propagation of the ischemic tissue damage as well as in mediating post-stroke regeneration. In this review, I discuss the functions of the miRNA miR-155 and the effect of its in vivo inhibition on brain recovery following experimental cerebral ischemia. The article introduces new and unexplored approach to cerebral regeneration: regulation of brain tissue repair through a direct modulation of specific miRNA activity.

Keywords: microRNA, miR-155, dMCAO, post-stroke inflammation, cerebral blood flow, functional recovery

\section{INTRODUCTION}

Stroke is a major public health problem in the United States, with 795,000 stroke survivors each year (610,000 of these are first attacks). According to the global stroke burden assessment, in 2013 there were more than 25 million stroke patients worldwide, with $\sim 10.3$ million having a first stroke. Ischemic strokes account for up to $71 \%$ of all stroke cases and $51 \%$ of all stroke-related deaths worldwide, and they are currently among the top leading causes of serious, long-term disability (Feigin et al., 2016). Stroke-associated ischemic damage involves cellular bioenergetic failure, excitotoxicity, oxidative stress, blood-brain barrier dysfunction, microvascular injury, post-ischemic inflammation, and, ultimately, the death of neurons, glia, and endothelial cells. The endothelial cell damage leads to a significant microvascular injury that directly contributes to cerebral tissue damage via increased endothelial cell permeability, matrix degradation, and the loss of cerebrovascular autoregulation (Brouns and De Deyn, 2009; Xing et al., 2012). The ischemic cascade leads to the development of the infarct core, which is represented by nonviable brain tissue. A hypoxic, but still viable, peri-infarct area surrounding the core region is a subject of intensive investigation, with a focus on neuroprotective and pro-regenerative treatments for preservation of salvageable brain tissue. The subacute phase of ischemia is accompanied by the active regeneration process, including post-ischemic angiogenesis and vasculogenesis in the ischemic boundary zone, which enhances oxygen and nutrient supply to the affected tissue (Ding et al., 2008; Beck and Plate, 2009). Stroke induces a potent neurogenic response and a massive migration of neural progenitors into the lesion area, which may substantially 
contribute to recovery and repair processes (Arvidsson et al., 2002; Kernie and Parent, 2010). In addition, cerebral ischemia triggers neuronal plasticity reflected in formation of new structural and functional connections between the neurons from the area adjacent to the infarcted tissue and the surrounding "healthy" brain tissue of the same hemisphere (Carmichael et al., 2017). Post-ischemic inflammatory response is an integral part of both brain damage and recovery. Post-ischemic elevation of cytokines is associated with recruitment of neutrophils, lymphocytes, and monocytes and activation of resident microglia, astrocytes, and endothelial cells. Activation of microglia and astrocytes leads to the additional release of pro-inflammatory factors (Liguz-Lecznar and Kossut, 2013). Lower levels of pro-inflammatory cytokines and higher expression of anti-inflammatory cytokines are associated with lower infarct size and a better clinical outcome (Perera et al., 2006; Lakhan et al., 2009). However, there is controversy surrounding the dual role of neuroinflammation in tissue damage and recovery: the post-stroke inflammation events contribute to brain injury but, on the other hand, could participate in tissue remodeling and recovery following brain damage. All of the described functionally-linked regeneration processes (including vascular remodeling, neural stem cell activation, neuronal plasticity, and neuroinflammation) reflect crosstalk between the components of the injured brain tissue. Molecular mechanisms of this complex repair process are extensively studied in search of possible targets for therapeutic intervention.

\section{Strategies to Improve a Post-stroke Recovery}

Despite the recent progress in post-stroke survival, therapeutic approaches directed toward recovery remain limited. Traditional approaches utilizing tissue-plasminogen activator (rt-PA) for thrombolysis are associated with time limitations (a 3-4 h therapeutic window) and possible complications (Lakhan et al., 2009). Neurorestorative processes may be enhanced by improving angiogenesis, neuroplasticity, and neurogenesis. Proposed approaches for vascular repair include the injection of angiogenesis-promoting compounds (Navaratna et al., 2009) or the transplantation of the endothelial progenitors (Fan et al., 2010; Bai et al., 2015) or neural stem/progenitor cells (Roitbak et al., 2008). Stem cell-based therapy for stroke and current pharmacological and tissue engineering approaches are discussed in detail in most recent reviews (Carmichael, 2016; Venkat et al., 2017).

All of the described therapeutic approaches are associated with certain limitations and complications, such as the increased vascular permeability and edema following VEGF treatment or possible transplant rejection, tumor formation, and infection associated with progenitor cell transplantation. An emerging and yet unexplored approach for treatment is based on the epigenetic processes associated with stroke (Pearce, 2011; Kassis et al., 2017). Among the epigenetic mechanisms that regulate stroke progression and recovery are the signaling pathways mediated by recently identified short non-coding RNAs called microRNAs.

\section{microRNAs}

miRNAs are a diverse class of highly conserved small RNA molecules that function as critical regulators of gene expression and are able to greatly influence cell development, differentiation, proliferation, and apoptosis (Kato and Slack, 2008). It is estimated that a large number of encoded genes are regulated by these single-stranded non-coding short (18-24 nucleotides long) RNAs. The miRNAs bind to their mRNA target at complementary sequences and downregulate gene expression by inhibiting the mRNA translation into proteins or by inducing mRNA degradation (Sun et al., 2010). The miRNAs control post-transcriptional gene expression in many tissues, including the brain (Ludwig et al., 2016). miRNA profiles have been characterized in the cerebral vasculature, neurons, astrocytes, and microglia (Kosik, 2006; Butovsky et al., 2014; Lopez-Ramirez et al., 2014; Rao et al., 2016).

Recent findings demonstrate that miRNAs orchestrate a variety of signaling pathways involved in stroke progression and post-stroke recovery, including neurogenesis (Zhao et al., 2008; Shi et al., 2010), endothelial cell morphogenesis (Poliseno et al., 2006; Suarez et al., 2007), and neuroinflammation (Gaudet et al., 2017). Multiple miRNAs have been found to play a profound role in stroke progression. The role of the miRNAs and the associated molecular mechanisms are discussed in detail in several excellent reviews (Lim et al., 2010; Vemuganti, 2010; Khoshnam et al., 2017; Li et al., 2017). Significant changes of miRNA profiles in the brain tissue and blood were detected at different times after the experimental ischemia in rodents (Liu et al., 2010). Numerous studies have reported a significant deregulation of specific circulating miRNAs in stroke patients (Jickling et al., 2014; Sepramaniam et al., 2014). These distinctive temporal changes are now regarded as indicators of the risk, occurrence, severity, and prognosis of stroke and have prompted an extensive search for specific peripheral miRNAs as biomarkers of the disease (Vijayan and Reddy, 2016). Another approach constitutes targeted regulation of the specific microRNA activity to stimulate a recovery process after stroke.

\section{In Vivo Regulation of miRNAs}

Systemic or local administration of the miRNA inhibitors and mimics are utilized to regulate the activity of specific miRNAs in various experimental animal models, including brain tumor (Teplyuk et al., 2016), breast cancer (Zhao et al., 2013), pancreatic cancer (Gibori et al., 2018), and pulmonary hypertension (McLendon et al., 2015) disease models. The first steps are performed to apply this technology to humans, such as subcutaneous delivery of anti-miR-122 in chronic hepatitis C patients (Stelma et al., 2017; van der Ree et al., 2017). This method could provide a future therapeutic potential in treatment of various diseases (Linnstaedt et al., 2010; Zhang et al., 2013). In vivo regulation of miRNAs in the animal models of stroke has been achieved by direct intracerebroventricular injections of specific antagomirs for miRNAs miR-493 and miR-23a-3p (Zhao et al., 2013; Li et al., 2016). Our recent investigations involving the in vivo inhibition of miRNA miR-155 introduced a novel approach to cerebral regeneration after stroke: regulation 
of post-stroke recovery via systemic injection of specific miRNA inhibitor.

\section{miR-155 Functions}

miR-155 is a multifunctional miRNA implicated in regulating various physiological and pathological processes such as hematopoietic lineage differentiation, immunity, inflammation, cancer, and cardiovascular diseases (Faraoni et al., 2009; O'Connell et al., 2010). miR-155 is specifically expressed in hematopoietic cells and cells involved in vascular remodeling, including B-cells, T-cells, monocytes, and granulocytes as well as endothelial cells and smooth muscle cells (Landgraf et al., 2007; Sun et al., 2012). Among other functions, miR-155 is involved in regulating the endothelial and vascular function (Sun et al., 2012; Weber et al., 2014). Downregulation of this miRNA is accompanied by reduced inflammation and improved regeneration processes (Kurowska-Stolarska et al., 2011; Murugaiyan et al., 2011; van Solingen et al., 2014). In addition to these multiple functions, miR-155 is involved in progression of multiple CNS disorders and pathological conditions. Some of the recent studies describing these miR-155 functions are summarized in Table 1.

\section{EFFECT OF SYSTEMIC MiR-155 INHIBITION AFTER THE EXPERIMENTAL CEREBRAL ISCHEMIA}

Our in vitro studies identified miR-155 as a potential regulator of the endothelial morphogenesis: specific miR-155 antisense inhibitors supported capillary-like tube formation by the mouse brain endothelial cells (Roitbak et al., 2011). We hypothesized that the inhibition of miR-155 after the experimental ischemia could support cerebral vasculature and improve vascular function. Intravenous injections of a specific anti-miR-155 inhibitor, initiated at $48 \mathrm{~h}$ after mouse distal middle cerebral artery occlusion (dMCAO), resulted in $~ 50 \%$ downregulation of miR-155 in the injured hemisphere of the mouse brain. In vivo two-photon laser scanning microscopy imaging and quantification of red blood cell (RBC) flow velocity detected a significantly improved blood flow in the peri-infarct area of the miR-155 inhibitor-injected mice during the first 2 weeks after dMCAO (Caballero-Garrido et al., 2015). These animals also demonstrated an improved vascular integrity and wellpreserved capillary tight junctions (TJs). An assessment of the brain tissue damage using MRI and electron microscopy (EM) demonstrated that at 3 weeks after stroke there was a significant $(34 \%)$ reduction of the infarct size and a significant decrease in neuronal damage in miR-155 inhibitor-injected animals, as compared to the control group. Improved TJ integrity in the inhibitor-injected animals was accompanied by the increased expression of major TJ protein ZO-1 and miR-155 target protein Rheb (Caballero-Garrido et al., 2015).

miR-155 inhibition after dMCAO significantly altered the time course and the expression levels of the major cytokines (including IL-10, IL-4, IL-6, MIP-1 $\alpha$, IL-5, and IL-17) as well

TABLE 1 | Involvement of miR-155 in CNS disorders and pathological conditions.

\section{Amyotrophic lateral} sclerosis (ALS)

Epilepsy

Stroke

Experimental autoimmune encephalomyelitis (EAE)

Spinal cord injury

Multiple sclerosis (MS)

Alzheimer's disease (AD)

Parkinson's disease (PD)

Brain tumor
Increased expression in CNS tissue from ALS patients and in mouse model of ALS (SOD1 mice). Inhibition prolongs survival and ameliorates disease in SOD1 mice (Koval et al., 2013; Butovsky et al., 2015).

Regulates glutamate uptake capacity of astrocytes in epilepsy (Gao et al., 2017). Significantly upregulated in the hippocampal tissues of children with Medial temporal lobe epilepsy (Ashhab et al., 2013).

Expression significantly affected by cerebral ischemia in rodents (Liu et al., 2010). Increased expression in rat model of stroke; inhibition supports post-stroke recovery (Caballero-Garrido et al., 2015; Xing et al., 2016).

Reduced severity of EAE in miR-155 KO mice (Murugaiyan et al., 2011). Knockdown results in reduction of Th1 and Th17 cells and mild EAE (Zhang et al., 2014).

Improved locomotor recovery in miR-155 KO mice (Yi et al., 2015; Gaudet et al., 2017).

Upregulated in blood and brain samples of MS patients (Lopez-Ramirez et al., 2014; Zhang et al., 2014).

Upregulation contributes to neuroinflammation in transgenic mouse model of AD (Guedes et al., 2014). Regulates T-lymphocyte activation and function in AD (Song and Lee, 2015).

Increased expression in mouse model of PD; mediates inflammatory responses (Thome et al., 2016).

Promotes glioma cell proliferation and contributes to glioma progression (Zhou et al., 2013; Yan et al., 2015); Increased expression in glioma tissue is associated with poor prognosis in patients (Sun et al., 2012). Upregulated in glioblastoma and promotes tumor growth (D'Urso et al., 2012).

as considerably modified the microglia/macrophage phenotype in the peri-infarct area of stroke. Electron microscopy-based quantification detected a decreased number of phagocytically active peri-vascular microglia/macrophages $(\mathrm{M} / \mathrm{Ms})$ in these animals (Pena-Philippides et al., 2016).

The assessment of sensorimotor deficits (bilateral asymmetry/adhesive removal test) and gait/locomotion recovery (CatWalk system), as well as the weight-gain evaluation, indicated that the inhibitor-injected animals regained their sensorimotor deficits and recovered faster than controls (Caballero-Garrido et al., 2015). Thus, miR155-inhibition-induced support of the cerebral vasculature, preservation of brain tissue, and the modified course of post-stroke neuroinflammation reflected in more efficient functional recovery of the inhibitor-injected animals. To our knowledge, this is among first reports describing the efficacy of intravenous antagomir injections performed during the sub-acute phase of the experimental stroke. Another study involving intravenous anti-miR-181 demonstrated improved animal recovery in mouse transient focal ischemia model (Xu et al., 2015). Experiments utilizing miR-155 inhibition in the 
rat model of cerebral ischemia supported our studies and demonstrated the efficacy of anti-miR-155 treatment in rats (Xing et al., 2016). In this study, the intracerebroventricular injections of anti-miR-155 performed at $24 \mathrm{~h}$ before the MCAO resulted in reduced infarct size and improved recovery. Similar to our findings, this effect was associated with the activation of miR-155 target Rheb (Xing et al., 2016).

\section{MOLECULAR MECHANISMS OF miR-155 INHIBITION-INDUCED SUPPORT OF POST-STROKE RECOVERY}

Possible mechanisms and consequences associated with in vivo inhibition of miR-155 after stroke are summarized in Figure 1. The improved blood supply to the peri-infarct area of the injured hemisphere detected in the inhibitor-injected animals could be mainly attributed to miR-155 inhibition-induced preservation of the endothelial TJs and thus BBB integrity. Based on the analyses, strengthening of TJs could be mediated by miR-155 inhibition-induced stabilization of ZO-1, a regulatory scaffolding protein critical for the TJ assembly and signal transduction (Antonetti et al., 1999; Harhaj and Antonetti, 2004; Balda and Matter, 2009). Since ZO-1 is not a direct target of miR-155, the exact mechanism of this regulation is unclear. Based on the analyses, miR-155 downregulation-induced ZO-1 stability could be mediated by the upregulation of a direct miR-155 target protein Rheb. Interestingly, stabilization of ZO-1 after miR-155 inhibition was also detected in human brain vascular endothelial cells in vitro (our unpublished data; Lopez-Ramirez et al., 2014). In these studies, the increased expression of ZO-1 and its stabilization on the endothelial cell membrane was associated with miR-155 direct target protein claudin-1. Identification of new miR-155 target proteins in the future could further clarify the effect of miR-155 activity on ZO-1 protein.

In the in vivo setting, other direct miR-155 targets upregulated in the inhibitor-injected mouse brain could broadly influence vascular function and brain tissue remodeling. These molecules are implicated in the regulation of angiogenesis, vascular stabilization and remodeling as well as vasodilation, neurovascular inflammation, and neuroprotection and thus could significantly contribute to the observed beneficial neurovascular effect of miR-155 inhibition (Figure 1). Detected elevated expression of SMAD5 protein may be associated with the activation of the bone morphogenetic protein (BMP) pathway, implicated in reduction of inflammation and vasculature stabilization (Marchuk et al., 2003; Pardali et al., 2010). Increased expression of Rictor, a major component of mTORC2 complex, could activate mTOR signaling, implicated in supporting angiogenesis (through activation of nitric oxide signaling) and neuroprotection after stroke (Karar and Maity, 2011; Chong et al., 2013). Endothelial nitric oxide synthase (eNOS) is associated with the improvement of blood flow and decreased rates of neuronal injury (Srivastava et al., 2012). Neuroprotective and overall pro-regenerative effect of miR-155 inhibition could be also mediated via its influence on brainderived neurotrophic factor (BDNF) expression (Varendi et al., 2014).

In addition to these possible mechanisms, downregulation of pro-inflammatory miR-155 could improve the recovery outcome by significantly influencing post-stroke inflammation. The effect of miR-155 silencing was characterized by a suppression of an early, transient, harmful increase of pro-inflammatory cytokines, followed by sustained upregulation of neuroprotective cytokines at the later stages of stroke recovery (14 days after stroke). At 7 days after dMCAO, in the anti-miR-155 injected animals, there was a decreased expression of pro-inflammatory cytokines CCL12 and CXCL3, implicated in vascular inflammation, accompanied by the upregulation of miR-155 direct targets and cytokine suppressors SHIP-1 and SOCS-1 as well as downregulation of JAK/STAT (Janus kinase/signal transducers and activators of transcription) pathway-mediated cytokine signaling. miR-155 inhibition resulted in a reduced number of phagocytic perivascular microglia/macrophages (M/Ms) and decreased expression of macrophage marker CD45 and active phagocytosis marker CD68 (Pena-Philippides et al., 2016). Based on these findings, we concluded that miR-155 induced repression of cytokine signaling and decreased $\mathrm{M} / \mathrm{M}$ phagocytic activity could contribute to preservation of TJs observed at 7 days after dMCAO.

At 14 days after dMCAO there was a sustained increase in expression of IL-10 in the inhibitor-injected animals. This major anti-inflammatory cytokine was shown to trigger an anti-inflammatory response beneficial for stroke outcome (Bazzoni et al., 2010; Perez-de Puig et al., 2013). High levels of IL-10 after the miR-155 inhibition could be induced by the observed prolonged elevation of miR-155 target and transcription factor C/EBP- $\beta$. Among other cytokines, with the increased expression at 14 days, were Il-4, IL-5, IL-6, and Il-17, which are characterized by context-dependent dual action and have a significant impact on neuroprotection and overall stroke outcome (Kim et al., 1995; Loddick et al., 1998; Li et al., 2001; Jiang et al., 2008; Erta et al., 2012). In contrast to 7 days, at 14 days after stroke there was an increase of CD45 and CD68positive $\mathrm{M} / \mathrm{Ms}$ in the peri-infarct area of miR-155 inhibited mice (Pena-Philippides et al., 2016). According to the literature, Iba1/CD45-positive macrophages expressing active phagocytosis marker CD68 facilitate the brain recovery process following stroke (Wattananit et al., 2016). Based on our data, we propose that miR-155 inhibition at $48 \mathrm{~h}$ after stroke results in suppression of early transient harmful actions of the activated M/Ms at 7 days, followed by an enhancement of their protective and reparative functions at 14 days after dMCAO.

\section{CONCLUSION}

Based on these findings, I conclude that systemic inhibition of miR-155 at $48 \mathrm{~h}$ following the experimental cerebral ischemia supports cerebral microvasculature and improves cerebral blood supply to the peri-infarct area of stroke at 7 days after stroke. These constructive processes following miR-155 downregulation 


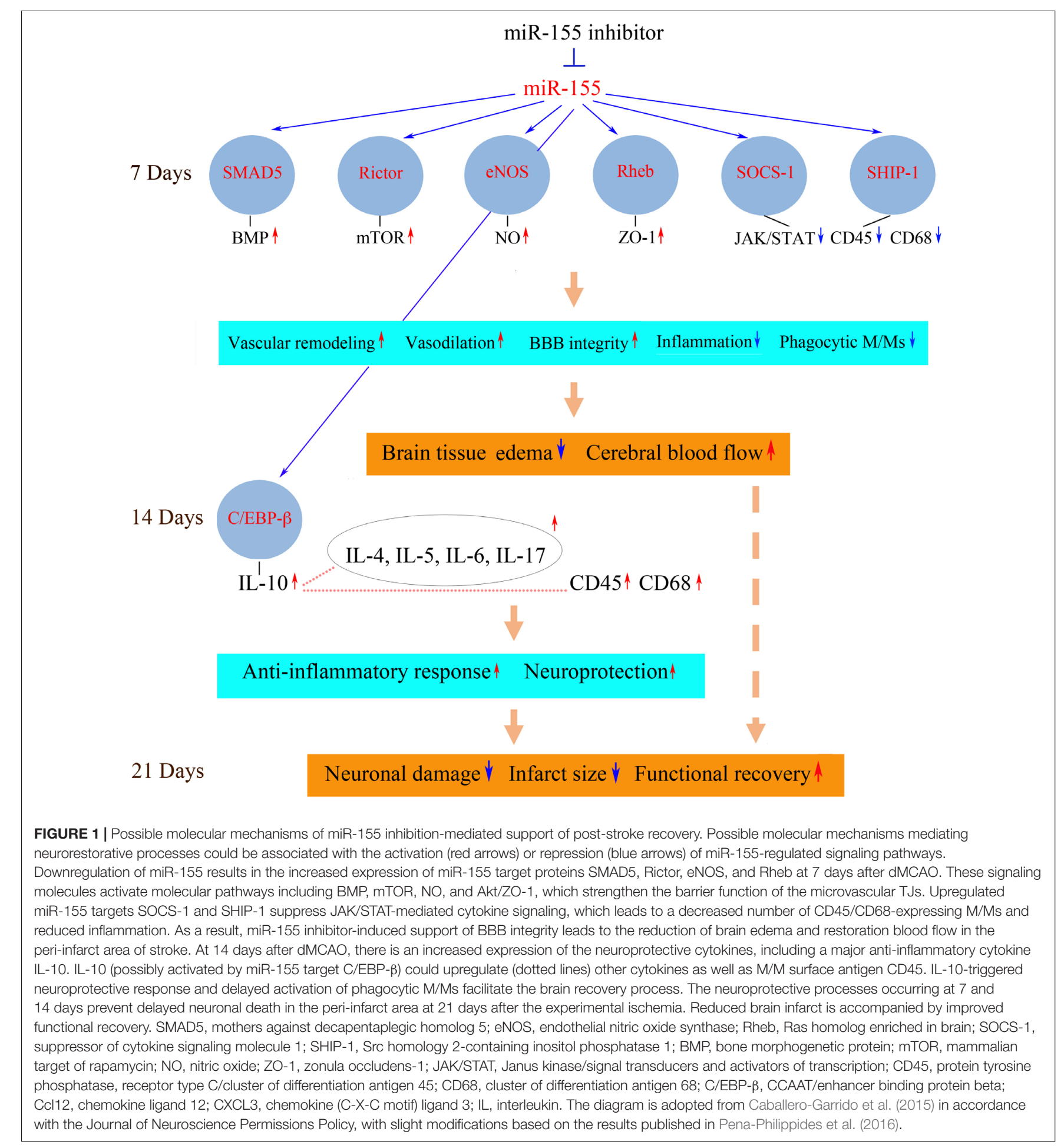

are achieved via direct preservation of TJ integrity and suppression of the early stage post-stroke inflammation. The vascular support at 7 days results in neuroprotection, reduction of the infarct size, and improvement in functional recovery at the later stages (21 days) of regeneration. This recovery mechanism is facilitated by: (1) the initial preservation of vascular integrity, which prevents the propagation of the ischemic damage into the peri-infarct area; (2) the activation of IL-10mediated neuroprotective mechanisms, and (3) transition from harmful phenotype toward the neuroprotective and reparative microglia/macrophage phenotype. All these support mechanisms could be mediated via the activation of miR-155 direct target proteins, including Rheb, SMAD5, Rictor, eNOS, SOCS-1, SHIP1 , and $\mathrm{C} / \mathrm{EBP}-\beta$. 


\section{CHALLENGES AND FUTURE IMPLICATIONS}

As we observed in our studies, silencing of a single miRNA can regulate a broad set of target genes and trigger synergistic therapeutic effect. Based on this advantage, in vivo regulation of miRNAs after stroke could become a promising approach to cerebral regeneration. One of the major challenges of miRNA-based therapy in patients is achieving specific, safe, and efficient regulation of particular miRNA expression. Systemic delivery of miRNA inhibitors and mimics is problematic because of their instability in blood circulation. Therefore, lipid-based delivery vehicles, viral vectors, and nanoparticle-conjugated oligonucleotides are utilized for the introduction of synthetic miRNA inhibitors and mimics in vivo. Recently developed Locked Nucleic Acid (LNA)-based technology (successfully used in our in vivo studies) greatly increases the affinity of the inhibitors for their target microRNAs, improves their resistance to enzymatic degradation, and minimizes off-target effects. Future innovations around the delivery techniques are expected, as miRNA-based treatment remains to be of great interest for the pharmacological industry. Delivery of the antisense inhibitors across the blood-brain barrier creates an additional challenge. This problem is minimized due to increased BBB leakage following stroke; however, impaired cerebral circulation could affect the inhibition efficacy.

Discovery of more stroke-associated miRNAs could lead to the development of a combinatory therapy involving the regulation of multiple miRNA expression: targeting a subset of genes with multiple miRNAs should enhance the therapeutic

\section{REFERENCES}

Antonetti, D. A., Barber, A. J., Hollinger, L. A., Wolpert, E. B., and Gardner, T. W. (1999). Vascular endothelial growth factor induces rapid phosphorylation of tight junction proteins occludin and zonula occluden 1 . A potential mechanism for vascular permeability in diabetic retinopathy and tumors. J. Biol. Chem. 274, 23463-23467. doi: 10.1074/jbc.274.33.23463

Arvidsson, A., Collin, T., Kirik, D., Kokaia, Z., and Lindvall, O. (2002). Neuronal replacement from endogenous precursors in the adult brain after stroke. Nat. Med. 8, 963-970. doi: 10.1038/nm747

Ashhab, M. U., Omran, A., Kong, H., Gan, N., He, F., Peng, J., et al. (2013). Expressions of tumor necrosis factor alpha and microRNA-155 in immature rat model of status epilepticus and children with mesial temporal lobe epilepsy. J. Mol. Neurosci. 51, 950-958. doi: 10.1007/s12031-0130013-9

Bai, Y. Y., Peng, X. G., Wang, L. S., Li, Z. H., Wang, Y. C., Lu, C. Q., et al. (2015). Bone marrow endothelial progenitor cell transplantation after ischemic stroke: an investigation into its possible mechanism. CNS Neurosci. Ther. 21, 877-886. doi: $10.1111 / \mathrm{cns} .12447$

Balda, M. S., and Matter, K. (2009). Tight junctions and the regulation of gene expression. Biochim. Biophys. Acta 1788, 761-767. doi: 10.1016/j.bbamem.2008. 11.024

Bazzoni, F., Tamassia, N., Rossato, M., and Cassatella, M. A. (2010). Understanding the molecular mechanisms of the multifaceted IL-10-mediated anti-inflammatory response: lessons from neutrophils. Eur. J. Immunol. 40, 2360-2368. doi: 10.1002/eji.200940294

Beck, H., and Plate, K. H. (2009). Angiogenesis after cerebral ischemia. Acta Neuropathol. 117, 481-496. doi: 10.1007/s00401-009-0483-6 effect. Certain caution should be given to possible side effects of these molecules in human trials, with specific emphasis on safety, tolerability, and efficacy of treatment. In fact, systemic miR-155 inhibition may influence immune function and negatively interfere with post-stroke recovery in humans. The optimal dose and time of the therapeutic intervention with specific microRNA inhibitors or mimics should be carefully determined to avoid any destructive intervention into the natural regeneration process. This therapeutic approach should be based on the knowledge of specific microRNA function, including its influence on the viability, proliferation, and differentiation of the brain tissue components as well as its possible effect on cerebral vasculature and blood flow, post-stroke inflammation, thrombosis, and atherosclerosis, etc. The degree of the inhibition or overexpression of specific miRNAs should also be taken into consideration; moderate systemic downregulation or upregulation could be a better choice to avoid secondary non-specific off-target effects or negative side effects such as impairment of the immune function.

\section{AUTHOR CONTRIBUTIONS}

The author confirms being the sole contributor of this work and approved it for publication.

\section{FUNDING}

This work was supported by the National Institute of Neurological Disorders and Stroke -NIH R01NS082225 grant.

Brouns, R., and De Deyn, P. P. (2009). The complexity of neurobiological processes in acute ischemic stroke. Clin. Neurol. Neurosurg. 111, 483-495. doi: 10.1016/j. clineuro.2009.04.001

Butovsky, O., Jedrychowski, M. P., Cialic, R., Krasemann, S., Murugaiyan, G., Fanek, Z., et al. (2015). Targeting miR-155 restores abnormal microglia and attenuates disease in SOD1 mice. Ann. Neurol. 77, 75-99. doi: 10.1002/ana. 24304

Butovsky, O., Jedrychowski, M. P., Moore, C. S., Cialic, R., Lanser, A. J., Gabriely, G., et al. (2014). Identification of a unique TGF-beta-dependent molecular and functional signature in microglia. Nat. Neurosci. 17, 131-143. doi: 10.1038/nn.3599

Caballero-Garrido, E., Pena-Philippides, J. C., Lordkipanidze, T., Bragin, D., Yang, Y., Erhardt, E. B., et al. (2015) In vivo inhibition of miR-155 promotes recovery after experimental mouse stroke. J. Neurosci. 35, 12446-12464. doi: 10.1523/JNEUROSCI.1641-15.2015

Carmichael, S. T. (2016). Emergent properties of neural repair: elemental biology to therapeutic concepts. Ann. Neurol. 79, 895-906. doi: 10.1002/ana. 24653

Carmichael, S. T., Kathirvelu, B., Schweppe, C. A., and Nie, E. H. (2017). Molecular, cellular and functional events in axonal sprouting after stroke. Exp. Neurol. 287(Pt 3), 384-394. doi: 10.1016/j.expneurol.2016.02.007

Chong, Z. Z., Yao, Q., and Li, H. H. (2013). The rationale of targeting mammalian target of rapamycin for ischemic stroke. Cell. Signal. 25, 1598-1607. doi: 10. 1016/j.cellsig.2013.03.017

Ding, G. L., Jiang, Q., Li, L., Zhang, L., Zhang, Z. G., Ledbetter, K. A., et al. (2008). Magnetic resonance imaging investigation of axonal remodeling and angiogenesis after embolic stroke in sildenafil-treated rats. J. Cerebr. Blood Flow Metab. 28, 1440-1448. doi: 10.1038/Jcbfm.2008.33 
D’Urso, P. I., D’Urso, O. F., Storelli, C., Mallardo, M., Gianfreda, C. D., Montinaro, A., et al. (2012). miR-155 is up-regulated in primary and secondary glioblastoma and promotes tumour growth by inhibiting GABA receptors. Int. J. Oncol. 41, 228-234. doi: 10.3892/ijo.2012.1420

Erta, M., Quintana, A., and Hidalgo, J. (2012). Interleukin-6, a major cytokine in the central nervous system. Int. J. Biol. Sci. 8, 1254-1266. doi: 10.7150/ijbs.4679

Fan, Y., Shen, F., Frenzel, T., Zhu, W., Ye, J., Liu, J., et al. (2010). Endothelial progenitor cell transplantation improves long-term stroke outcome in mice. Ann. Neurol. 67, 488-497. doi: 10.1002/ana.21919

Faraoni, I., Antonetti, F. R., Cardone, J., and Bonmassar, E. (2009). miR-155 gene: a typical multifunctional microRNA. Biochim. Biophys. Acta 1792, 497-505. doi: 10.1016/j.bbadis.2009.02.013

Feigin, V. L., Norrving, B., George, M. G., Foltz, J. L., Roth, G. A., and Mensah, G. A. (2016). Prevention of stroke: a strategic global imperative. Nat. Rev. Neurol. 12, 501-512. doi: 10.1038/nrneurol.2016.107

Gao, W., Bi, Y., Ding, L., Zhu, W., and Ye, M. (2017). SSa ameliorates the Glu uptaking capacity of astrocytes in epilepsy via AP-1/miR-155/GLAST. Biochem. Biophys. Res. Commun. 493, 1329-1335. doi: 10.1016/j.bbrc.2017.09.139

Gaudet, A. D., Fonken, L. K., Watkins, L. R., Nelson, R. J., and Popovich, P. G. (2017). MicroRNAs: roles in regulating neuroinflammation. Neuroscientist doi: 10.1177/1073858417721150 [Epub ahead of print].

Gibori, H., Eliyahu, S., Krivitsky, A., Ben-Shushan, D., Epshtein, Y., Tiram, G., et al. (2018). Amphiphilic nanocarrier-induced modulation of PLK1 and miR34 a leads to improved therapeutic response in pancreatic cancer. Nat. Commun. 9:16. doi: 10.1038/s41467-017-02283-9

Guedes, J. R., Custodia, C. M., Silva, R. J., de Almeida, L. P., Pedroso de Lima, M. C., Cardoso, A. L., et al. (2014). Early miR-155 upregulation contributes to neuroinflammation in Alzheimer's disease triple transgenic mouse model. Hum. Mol. Genet. 23, 6286-6301. doi: 10.1093/hmg/ddu348

Harhaj, N. S., and Antonetti, D. A. (2004). Regulation of tight junctions and loss of barrier function in pathophysiology. Int. J. Biochem. Cell Biol. 36, 1206-1237. doi: 10.1016/j.biocel.2003.08.007

Jiang, L., Newman, M., Saporta, S., Chen, N., Sanberg, C., Sanberg, P. R., et al. (2008). MIP-1alpha and MCP-1 induce migration of human umbilical cord blood cells in models of stroke. Curr. Neurovasc. Res. 5, 118-124. doi: 10.2174/ 156720208784310259

Jickling, G. C., Ander, B. P., Zhan, X., Noblett, D., Stamova, B., and Liu, D. (2014). microRNA expression in peripheral blood cells following acute ischemic stroke and their predicted gene targets. PLoS One 9:e99283. doi: 10.1371/journal.pone. 0099283

Karar, J., and Maity, A. (2011). PI3K/AKT/mTOR pathway in Angiogenesis. Front. Mol. Neurosci. 4:51. doi: 10.3389/fnmol.2011.00051

Kassis, H., Shehadah, A., Chopp, M., and Zhang, Z. G. (2017). Epigenetics in stroke recovery. Genes 8:89. doi: 10.3390/genes 8030089

Kato, M., and Slack, F. J. (2008). microRNAs: small molecules with big roles C. elegans to human cancer. Biol. Cell 100, 71-81. doi: 10.1042/BC20070078

Kernie, S. G., and Parent, J. M. (2010). Forebrain neurogenesis after focal Ischemic and traumatic brain injury. Neurobiol. Dis. 37, 267-274. doi: 10.1016/j.nbd. 2009.11.002

Khoshnam, S. E., Winlow, W., and Farzaneh, M. (2017). The interplay of MicroRNAs in the inflammatory mechanisms following ischemic stroke. J. Neuropathol. Exp. Neurol. 76, 548-561. doi: 10.1093/jnen/nlx036

Kim, J. S., Gautam, S. C., Chopp, M., Zaloga, C., Jones, M. L., Ward, P. A., et al. (1995). Expression of monocyte chemoattractant protein-1 and macrophage inflammatory protein-1 after focal cerebral ischemia in the rat. J. Neuroimmunol. 56, 127-134. doi: 10.1016/0165-5728(94)00138-E

Kosik, K. S. (2006). The neuronal microRNA system. Nat. Rev. Neurosci. 7, 911-920. doi: 10.1038/nrn2037

Koval, E. D., Shaner, C., Zhang, P., du Maine, X., Fischer, K., Tay, J., et al. (2013). Method for widespread microRNA-155 inhibition prolongs survival in ALS-model mice. Hum. Mol. Genet. 22, 4127-4135. doi: 10.1093/hmg/ddt261

Kurowska-Stolarska, M., Alivernini, S., Ballantine, L. E., Asquith, D. L., Millar, N. L., Gilchrist, D. S., et al. (2011). MicroRNA-155 as a proinflammatory regulator in clinical and experimental arthritis. Proc. Natl. Acad. Sci. U.S.A. 108, 11193-11198. doi: 10.1073/pnas.1019536108

Lakhan, S. E., Kirchgessner, A., and Hofer, M. (2009). Inflammatory mechanisms in ischemic stroke: therapeutic approaches. J. Transl. Med. 7:97. doi: 10.1186/ 1479-5876-7-97
Landgraf, P., Rusu, M., Sheridan, R., Sewer, A., Iovino, N., Aravin, A., et al. (2007). A mammalian microRNA expression atlas based on small RNA library sequencing. Cell 129, 1401-1414. doi: 10.1016/j.cell.2007.04.040

Li, G., Morris-Blanco, K. C., Lopez, M. S., Yang, T., Zhao, H., Vemuganti, R., et al. (2017). Impact of microRNAs on ischemic stroke: from pre- to post-disease. Prog. Neurobiol. doi: 10.1016/j.pneurobio.2017.08.002 [Epub ahead of print].

Li, H. L., Kostulas, N., Huang, Y. M., Xiao, B. G., van der Meide, P., Kostulas, V., et al. (2001). IL-17 and IFN-gamma mRNA expression is increased in the brain and systemically after permanent middle cerebral artery occlusion in the rat. J. Neuroimmunol. 116, 5-14. doi: 10.1016/S0165-5728(01)00264-8

Li, Q., He, Q., Baral, S., Mao, L., Li, Y., Jin, H., et al. (2016). MicroRNA-493 regulates angiogenesis in a rat model of ischemic stroke by targeting MIF. FEBS J. 283, 1720-1733. doi: 10.1111/febs.13697

Liguz-Lecznar, M., and Kossut, M. (2013). Influence of inflammation on poststroke plasticity. Neural Plast. 2013:258582. doi: 10.1155/2013/258582

Lim, K. Y., Chua, J. H., Tan, J. R., Swaminathan, P., Sepramaniam, S., Armugam, A., et al. (2010). MicroRNAs in cerebral ischemia. Transl. Stroke Res. 1, 287-303. doi: 10.1007/s12975-010-0035-3

Linnstaedt, S. D., Gottwein, E., Skalsky, R. L., Luftig, M. A., and Cullen, B. R. (2010). Virally induced cellular microRNA miR-155 plays a key role in B-cell immortalization by Epstein-Barr virus. J. Virol. 84, 11670-11678. doi: 10.1128/ JVI.01248-10

Liu, D. Z., Tian, Y., Ander, B. P., Xu, H., Stamova, B. S., Zhan, X., et al. (2010). Brain and blood microRNA expression profiling of ischemic stroke, intracerebral hemorrhage, and kainate seizures. J. Cereb. Blood Flow Metab. 30, 92-101. doi: 10.1038/jcbfm.2009.186

Loddick, S. A., Turnbull, A. V., and Rothwell, N. J. (1998). Cerebral interleukin-6 is neuroprotective during permanent focal cerebral ischemia in the rat. J. Cereb. Blood Flow Metab. 18, 176-179. doi: 10.1097/00004647-199802000-00008

Lopez-Ramirez, M. A., Wu, D., Pryce, G., Simpson, J. E., Reijerkerk, A., KingRobson, J., et al. (2014). MicroRNA-155 negatively affects blood-brain barrier function during neuroinflammation. FASEB J. 28, 2551-2565. doi: 10.1096/fj. 13-248880

Ludwig, N., Leidinger, P., Becker, K., Backes, C., Fehlmann, T., Pallasch, C., et al. (2016). Distribution of miRNA expression across human tissues. Nucleic Acids Res. 44, 3865-3877. doi: 10.1093/nar/gkw116

Marchuk, D. A., Srinivasan, S., Squire, T. L., and Zawistowski, J. S. (2003). Vascular morphogenesis: tales of two syndromes. Hum. Mol. Genet. 12, R97-R112. doi: $10.1093 / \mathrm{hmg} / \mathrm{ddg} 103$

McLendon, J. M., Joshi, S. R., Sparks, J., Matar, M., Fewell, J. G., Abe, K., et al. (2015). Lipid nanoparticle delivery of a microRNA-145 inhibitor improves experimental pulmonary hypertension. J. Control. Release 210, 67-75. doi: 10.1016/j.jconrel.2015.05.261

Murugaiyan, G., Beynon, V., Mittal, A., Joller, N., and Weiner, H. L. (2011). Silencing microRNA-155 ameliorates experimental autoimmune encephalomyelitis. J. Immunol. 187, 2213-2221. doi: 10.4049/jimmunol. 1003952

Navaratna, D., Guo, S., Arai, K., and Lo, E. H. (2009). Mechanisms and targets for angiogenic therapy after stroke. Cell Adh. Migr. 3, 216-223. doi: 10.4161/cam.3. 2.8396

O’Connell, R. M., Kahn, D., Gibson, W. S., Round, J. L., Scholz, R. L., Chaudhuri, A. A., et al. (2010). MicroRNA-155 promotes autoimmune inflammation by enhancing inflammatory $\mathrm{T}$ cell development. Immunity 33, 607-619. doi: 10.1016/j.immuni.2010.09.009

Pardali, E., Goumans, M. J., and ten Dijke, P. (2010). Signaling by members of the TGF-beta family in vascular morphogenesis and disease. Trends Cell Biol. 20, 556-567. doi: 10.1016/j.tcb.2010.06.006

Pearce, W. J. (2011). Epigenetics: an expanding new piece of the stroke puzzle. Transl. Stroke Res. 2, 243-247. doi: 10.1007/s12975-011-0094-0

Pena-Philippides, J. C., Caballero-Garrido, E., Lordkipanidze, T., and Roitbak, T. (2016) In vivo inhibition of miR-155 significantly alters post-stroke inflammatory response. J. Neuroinflammation 13:287. doi: 10.1186/s12974-016-0753-x

Perera, M. N., Ma, H. K., Arakawa, S., Howells, D. W., Markus, R., Rowe, C. C., et al. (2006). Inflammation following stroke. J. Clin. Neurosci. 13, 1-8. doi: 10.1016/J.Jocn.2005.07.005

Perez-de Puig, I., Miro, F., Salas-Perdomo, A., Bonfill-Teixidor, E., FerrerFerrer, M., Marquez-Kisinousky, L., et al. (2013). IL-10 deficiency exacerbates 
the brain inflammatory response to permanent ischemia without preventing resolution of the lesion. J. Cereb. Blood Flow Metab. 33, 1955-1966. doi: $10.1038 /$ jcbfm.2013.155

Poliseno, L., Tuccoli, A., Mariani, L., Evangelista, M., Citti, L., Woods, K., et al. (2006). MicroRNAs modulate the angiogenic properties of HUVECs. Blood 108, 3068-3071. doi: 10.1182/blood-2006-01-012369

Rao, V. T., Ludwin, S. K., Fuh, S. C., Sawaya, R., Moore, C. S., Ho, M. K., et al. (2016). MicroRNA expression patterns in human astrocytes in relation to anatomical location and age. J. Neuropathol. Exp. Neurol. 75, 156-166. doi: 10.1093/jnen/nlv016

Roitbak, T., Bragina, O., Padilla, J. L., and Pickett, G. G. (2011). The role of microRNAs in neural stem cell-supported endothelial morphogenesis. Vasc. Cell 3:25. doi: 10.1186/2045-824X-3-25

Roitbak, T., Li, L., and Cunningham, L. A. (2008). Neural stem/progenitor cells promote endothelial cell morphogenesis and protect endothelial cells against ischemia via HIF-1alpha-regulated VEGF signaling. J. Cereb. Blood Flow Metab. 28, 1530-1542. doi: 10.1038/jcbfm.2008.38

Sepramaniam, S., Tan, J. R., Tan, K. S., DeSilva, D. A., Tavintharan, S., Woon, F. P., et al. (2014). Circulating microRNAs as biomarkers of acute stroke. Int. J. Mol. Sci. 15, 1418-1432. doi: 10.3390/ijms15011418

Shi, Y., Zhao, X., Hsieh, J., Wichterle, H., Impey, S., Banerjee, S., et al. (2010). MicroRNA regulation of neural stem cells and neurogenesis. J. Neurosci. 30, 14931-14936. doi: 10.1523/JNEUROSCI.4280-10.2010

Song, J., and Lee, J. E. (2015). miR-155 is involved in Alzheimer's disease by regulating T lymphocyte function. Front. Aging Neurosci. 7:61. doi: 10.3389/ fnagi.2015.00061

Srivastava, K., Bath, P. M., and Bayraktutan, U. (2012). Current therapeutic strategies to mitigate the eNOS dysfunction in ischaemic stroke. Cell Mol. Neurobiol. 32, 319-336. doi: 10.1007/s10571-011-9777-z

Stelma, F., van der Ree, M. H., Sinnige, M. J, Brown, A., Swadling, L., de Vree, J. M. L., et al. (2017). Immune phenotype and function of natural killer and $\mathrm{T}$ cells in chronic hepatitis $\mathrm{C}$ patients who received a single dose of anti-MicroRNA-122, RG-101. Hepatology 66, 57-68. doi: 10.1002/hep.29148

Suarez, Y., Fernandez-Hernando, C., Pober, J. S., and Sessa, W. C. (2007). Dicer dependent microRNAs regulate gene expression and functions in human endothelial cells. Circ. Res. 100, 1164-1173. doi: 10.1161/01.RES.0000265065 26744.17

Sun, H. X., Zeng, D. Y., Li, R. T., Pang, R. P., Yang, H., Hu, Y. L., et al. (2012). Essential role of microRNA-155 in regulating endothelium-dependent vasorelaxation by targeting endothelial nitric oxide synthase. Hypertension 60, 1407-1414. doi: 10.1161/HYPERTENSIONAHA.112.197301

Sun, W., Julie Li, Y. S., Huang, H. D., Shyy, J. Y., and Chien, S. (2010). microRNA: a master regulator of cellular processes for bioengineering systems. Annu. Rev. Biomed. Eng. 12:1-27. doi: 10.1146/annurev-bioeng-070909-105314

Teplyuk, N. M., Uhlmann, E. J., Gabriely, G., Volfovsky, N., Wang, Y., Teng, J., et al. (2016). Therapeutic potential of targeting microRNA-10b in established intracranial glioblastoma: first steps toward the clinic. EMBO Mol. Med. 8, 268-287. doi: 10.15252/emmm.201505495

Thome, A. D., Harms, A. S., Volpicelli-Daley, L. A., and Standaert, D. G. (2016). microRNA-155 regulates alpha-synuclein-induced inflammatory responses in models of parkinson disease. J. Neurosci. 36, 2383-2390. doi: 10.1523/ JNEUROSCI.3900-15.2016

van der Ree, M. H., de Vree, J. M., Stelma, F., Willemse, S., van der Valk, M., Rietdijk, S., et al. (2017). Safety, tolerability, and antiviral effect of RG-101 in patients with chronic hepatitis C: a phase $1 \mathrm{~B}$, double-blind, randomised controlled trial. Lancet 389, 709-717. doi: 10.1016/S0140-6736(16) 31715-9

van Solingen, C., Araldi, E., Chamorro-Jorganes, A., Fernandez-Hernando, C., and Suarez, Y. (2014). Improved repair of dermal wounds in mice lacking microRNA-155. J. Cell. Mol. Med. 18, 1104-1112. doi: 10.1111/jcmm. 12255

Varendi, K., Kumar, A., Harma, M. A., and Andressoo, J. O. (2014). miR-1, miR10b, miR-155, and miR-191 are novel regulators of BDNF. Cell. Mol. Life Sci. 71, 4443-4456. doi: 10.1007/s00018-014-1628-x
Vemuganti, R. (2010) The micrornas and stroke: no need to be coded to be counted. Transl. Stroke Res. 1, 158-160. doi: 10.1007/s12975-010-0030-8

Venkat, P., Shen, Y., Chopp, M., and Chen, J. (2017). Cell-based and pharmacological neurorestorative therapies for ischemic stroke. Neuropharmacology doi: 10.1016/j.neuropharm.2017.08.036 [Epub ahead of print].

Vijayan, M., and Reddy, P. H. (2016). Peripheral biomarkers of stroke: focus on circulatory microRNAs. Biochim. Biophys. Acta 1862, 1984-1993. doi: 10.1016/ j.bbadis.2016.08.003

Wattananit, S., Tornero, D., Graubardt, N., Memanishvili, T., Monni, E., Tatarishvili, J., et al. (2016). Monocyte-derived macrophages contribute to spontaneous long-term functional recovery after stroke in mice. J. Neurosci. 36, 4182-4195. doi: 10.1523/JNEUROSCI.4317-15.2016

Weber, M., Kim, S., Patterson, N., Rooney, K., and Searles, C. D. (2014). MiRNA-155 targets myosin light chain kinase and modulates actin cytoskeleton organization in endothelial cells. Am. J. Physiol. Heart Circ. Physiol. 306, H1192-H1203. doi: 10.1152/ajpheart.00521.2013

Xing, C., Arai, K., Lo, E. H., and Hommel, M. (2012). Pathophysiologic cascades in ischemic stroke. Int. J. Stroke 7, 378-385. doi: 10.1111/j.1747-4949.2012.00839.x

Xing, G., Luo, Z., Zhong, C., Pan, X., and Xu, X. (2016). Influence of miR155 on cell apoptosis in rats with ischemic stroke: role of the Ras Homolog Enriched in Brain (Rheb)/mTOR pathway. Med. Sci. Monit. 22, 5141-5153. doi: 10.12659/MSM.898980

Xu, L. J., Ouyang, Y. B., Xiong, X., Stary, C. M., and Giffard, R. G. (2015). Poststroke treatment with miR-181 antagomir reduces injury and improves longterm behavioral recovery in mice after focal cerebral ischemia. Exp. Neurol. 264:1-7. doi: 10.1016/j.expneurol.2014.11.007

Yan, Z., Che, S., Wang, J., Jiao, Y., Wang, C., and Meng, Q. (2015). miR155 contributes to the progression of glioma by enhancing Wnt/beta-catenin pathway. Tumour Biol. 36, 5323-5331. doi: 10.1007/s13277-015-3193-9

Yi, J., Wang, D., Niu, X., Hu, J., Zhou, Y., and Li, Z. (2015). microRNA155 deficiency suppresses Th17 cell differentiation and improves locomotor recovery after spinal cord injury. Scand. J. Immunol. 81, 284-290. doi: 10.1111/ sji. 12276

Zhang, J., Cheng, Y., Cui, W., Li, M., Li, B., and Guo, L. (2014). MicroRNA-155 modulates Th1 and Th17 cell differentiation and is associated with multiple sclerosis and experimental autoimmune encephalomyelitis. J. Neuroimmunol. 266, 56-63. doi: 10.1016/j.jneuroim.2013.09.019

Zhang, M., Zhou, X., Wang, B., Yung, B. C., Lee, L. J., Ghoshal, K., et al. (2013). Lactosylated gramicidin-based lipid nanoparticles (Lac-GLN) for targeted delivery of anti-miR-155 to hepatocellular carcinoma. J. Control. Release 168, 251-261. doi: 10.1016/j.jconrel.2013.03.020

Zhao, D., Tu, Y., Wan, L., Bu, L., Huang, T., Sun, X., et al. (2013) In vivo monitoring of angiogenesis inhibition via down-regulation of mir-21 in a VEGFR2-luc murine breast cancer model using bioluminescent imaging. PLoS One 8:e71472. doi: 10.1371/journal.pone.0071472

Zhao, T., Zhang, C. P., Liu, Z. H., Wu, L. Y., Huang, X., Wu, H. T., et al. (2008). Hypoxia-driven proliferation of embryonic neural stem/progenitor cells-role of hypoxia-inducible transcription factor-1alpha. FEBS J. 275, 1824-1834. doi: 10.1111/j.1742-4658.2008.06340.x

Zhou, J., Wang, W., Gao, Z., Peng, X., Chen, X., Chen, W., et al. (2013). MicroRNA155 promotes glioma cell proliferation via the regulation of MXI1. PLoS One 8:e83055. doi: 10.1371/journal.pone.0083055

Conflict of Interest Statement: The author declares that the research was conducted in the absence of any commercial or financial relationships that could be construed as a potential conflict of interest.

Copyright (C) 2018 Roitbak. This is an open-access article distributed under the terms of the Creative Commons Attribution License (CC BY). The use, distribution or reproduction in other forums is permitted, provided the original author $(s)$ and the copyright owner are credited and that the original publication in this journal is cited, in accordance with accepted academic practice. No use, distribution or reproduction is permitted which does not comply with these terms. 